\title{
Interference with McCollough effects via pre- and postinduction exposure to achromatic gratings: Time course and magnitude of aftereffect decrement
}

\author{
DIANE SKOWBO \\ Colby College, Waterville, Maine
}

\begin{abstract}
The strength of McCollough effects (MEs) may be reduced by having subjects view achromatic gratings either before or after ME induction. Both types of interference effects were investigated in a series of experiments designed to describe and compare their properties. Among the results were the following major findings: (1) Recovery of ME strength following postinduction interference manifests itself as a convergence of the interference decay curve and a control curve; it occurs within 30-60 min, and may or may not involve an increase in $\mathrm{ME}$ strength in the interference curve. (2) Preinduction interference does not occur if more than a few minutes separate the achromatic and chromatic inspections; interference effects that occur when the interinspection interval is zero are evident throughout the decay of the subsequent ME. These results are discussed in terms of several explanations of interference effects.
\end{abstract}

Aftereffects of apparent color that are contingent on spatial features of lined patterns were first reported by McCollough in 1965 and now are often known as McCollough effects (MEs) or as contingent aftereffects. They are typically induced by having subjects view two alternating patterns that contain complementary hues and orthogonal spatial features, for example, a pattern of black vertical lines on a green background and one of black horizontal lines on a red background. After a subject has viewed the patterns for several minutes, achromatic lined patterns appear tinted with weakly saturated hues approximately complementary to those previously associated with each spatial feature. In the example above, a pinkish color would appear on the white portions of a black and white vertical grating, and the white portions of a horizontal grating would have a greenish appearance.

One of the most striking characteristics of MEs is their longevity. Unlike aftereffects of color, such as afterimages, MEs typically persist for hours or days (e.g., Riggs, White, \& Eimas, 1974). Several investigators (e.g., Shute, 1979) have described the time course of ME decay with a function that drops rapidly at first and then more gradually.

A normal decay process may be arrested or accelerated by manipulations of the visual environment. For example, MacKay and MacKay (1975) reported that the ME did not decay in a totally occluded eye, but did decay in an eye receiving unpatterned, diffuse light. On the other

This research was supported by Colby College. I thank Marie Chadeayne, Susan Kaplan, Liz Kotler, and Henrie Rand for serving as subjects. Requests for reprints should be sent to Diane Skowbo, Department of Psychology, Colby College, Waterville, ME 04901. hand, MEs can be made to show an unusually rapid decline by having subjects view achromatic patterns with the same spatial features as the induction stimuli. This type of interference with the natural decay of MEs was first described by Skowbo, Gentry, Timney, and Morant (1974), whose experiment compared the effects of several types of post-ME stimulation; they found that MEs declined much more sharply after exposure to achromatic gratings than after exposure to other visual stimuli. In attempting to explain this finding, the authors speculated that MEs might be considered conditioned responses, with the rapid decay following the achromatic grating exposure akin to extinction.

Two years later, interference effects were investigated in an experiment described by Graves (1976) in her senior honors thesis at Brown and reported to the Association for Research in Vision and Ophthalmology (White \& Graves, 1976). This experiment was conducted to determine whether the magnitude of this interference effect varied with the length of time between chromatic and achromatic inspections. ME assessments carried out over a period of several hours revealed about the same amount of reduction regardless of the duration of the interinspection interval; furthermore, after an initial depression, there was a partial recovery of ME strength before further decline. White and Graves also reported that if the achromatic gratings were viewed before $\mathrm{ME}$ induction, the $\mathrm{ME}$ was weaker than in a control condition in which there was no prior exposure. Graves (1976) speculated that viewing achromatic gratings produced an independent aftereffect, possibly a reduction in apparent contrast of subsequently viewed induction or test patterns, which weakened their efficiency as visual stimuli. This argument was supported by subsequent evidence that MEs were less 
vivid on low-contrast test patterns than on high-contrast figures (White \& Graves, 1977).

The phenomena of preinduction interference and partial recovery from postinduction interference were explored in subsequent papers by Skowbo and others. The independent variables in Skowbo and Clynes's (1977) study were length of exposure to postinduction achromatic gratings and length of rest period before testing for recovery. Results indicated that longer achromatic grating exposure times produced more ME depression. No systematic differences in the extent of recovery were associated with rest-period times, but most recovery occurred following shortest achromatic exposure times. Two major explanations were considered. One was a learning account in which postinduction interference was interpreted as extinction and preinduction interference was considered to be latent inhibition. According to this line of thought, the partial gains in strength would be interpreted as spontaneous recovery. The other explanation posited the existence of an achromatic analogue to MEs, which combined with the chromatic effect to alter its assessed strength.

In 1979, Skowbo studied achromatic grating luminance as a factor in the interference effect. Three luminance levels were used; in both preinduction and postinduction effects, the gratings of highest luminance produced the most interference. The apparent contrast reduction hypothesis was considered to be generally supported by these results, but, on the other hand, it was noted that even a very low-luminance pattern, which should not produce much contrast reduction, reduced the ME to some $60 \%$ of its prior strength. Furthermore, the difference in amount of interference produced by the brightest versus the dimmest pattern (about 15\%) was small in comparison with the difference in luminance (about $2 \log$ units).

Skowbo (1979) evaluated the ability of the conditioning account of MEs to account for her results in the context of findings by White (1976) that MEs are strongest when built up with high-intensity grating patterns and when evaluated on low-intensity grating patterns. In Skowbo's experiment, the greatest amount of "extinction' was produced by patterns that, according to White's research, would evoke the weakest "conditioned response"; this seemed contrary to what would be expected. However, the results were considered to be consistent with a hypothesized achromatic ME that biased the visual system toward neutrality, rather than chromaticity; if an effect such as this summated with the chromatic one, it could reduce its apparent strength. Skowbo stressed that this explanation was extremely speculative, given that such effects had never been demonstrated independently.

An attempt to carry out such a demonstration was undertaken by White and Skowbo (1980). They reasoned that if an achromatic ME did exist, it would have a manifestation opposite that of the chromatic one, namely, a reduction in the saturation of actual hues. White and Skowbo constructed grating patterns with weakly saturated chromatic backgrounds that resembled MEs. Subjects matched the color appearance of these patterns be- fore and after viewing achromatic gratings; however, the matches were not affected by the achromatic inspections. The results of this experiment thus failed to support the notion that an independent achromatic aftereffect was the basis of interference.

The most recent experiment on interference phenomena was carried out by Savoy (1984), who induced in his subjects a binocular ME, and then had them view achromatic gratings monocularly. Subsequently, MEs in the two eye: were compared: the strength of the effect in the eye exposed to the achromatic gratings was markedly decreasec relative to that in the control eye. Savoy interpreted hi: findings primarily in the context of models that describe the ME in terms of interactions between color system: and achromatic spatial systems. However, he also discussed the possibility that interference effects reflect : hypothetical process he called "competition," whereby "adaptation to achromatic gratings builds up MEs for $a l$. colors, thus diluting the previously established effects" (p. 575).

Given the lack of agreement on the way in which ME: themselves are generated, it is perhaps not surprising tha there is little agreement on the mechanism by which achromatic gratings interfere with MEs. From the foregoin summary, it is evident that each of the explanations has a shortcoming. The extinction explanation presuppose. that MEs are themselves conditioned responses, a notion that has been vigorously debated in the literature (see e.g., Allan \& Siegel, 1986; Skowbo, 1986). In the opinion of Skowbo (1984), the aspects of MEs that mos closely resemble conditioning phenomena-postinductior interference (extinction?), the subsequent partial reviva (spontaneous recovery?), and preinduction interference (latent inhibition?)-all have in common the inspectior of achromatic gratings, which suggests the possibility c an alternative hypothesis dealing with that factor per se An attractive alternative hypothesis, however, is not avail able: the rather small effect of large variations in the lu minance of interference patterns detracts from the con trast reduction account; ideas that exposure to achromatir gratings generates another contingent aftereffect that sum mates or competes with the original ME have either failer or not been put to independent test.

Additional empirical work may be necessary as a bas for theory; careful examination of this literature suggest: that some characteristics of interference effects may no have been described in a maximally useful fashion. Non of the above-reviewed experiments had a control condi tion in which pre- or postinduction visual stimulation wa the same as in the interference condition except for th presence of contours. For example, in Skowbo et al (1974), the various inspection conditions differed in hue luminance, and spatial features; in White and Grave. (1976), the comparison curve described ME decay in thı absence of any additional stimulation; in Skowbo (1979) the effects of achromatic grating inspection were describe only in terms of their impact on a prior or on a typica ME. Descriptions of interference effects made in com 
parison with the effects of a matched-luminance control stimulus would enable us to obtain unequivocal answers to questions about the magnitude and time course of postinduction interference and recovery. For example, the question of how much recovery occurs should be answered in terms of the strength the ME would otherwise have had at a given point in time. Skowbo and Clynes (1977) answered this question only in terms of fractions of the original ME's strength, and, although data described by White and Graves (1976) suggest that a ME recovering from interference remains weaker throughout its decay than it normally would have been, as noted above, their comparison condition contained no postinduction control inspections.

There are also some unanswered questions that involve comparisons between preinduction and postinduction interference: are their magnitudes and time courses comparable? Can anything like recovery be observed following preinduction interference?

The experiments that follow were designed to answer these questions. They differ from most previous work on interference effects in that the luminance and duration of the achromatic stimuli are comparable to those of the patterns used to induce MEs.

\section{EXPERIMENT 1}

Experiment 1 was designed to provide a description of preinduction interference. Either the achromatic stimuli were presented immediately before the ME induction patterns, or there was a delay between the achromatic exposure and the ME induction.

\section{Method}

Subjects. Three females participated. According to their results on the Farnsworth-Munsell 100-Hue Test, each had colordiscriminating ability in the superior range. Before the experiment began, they acquired a number of MEs and practiced matching the resultant subjective hues with the color-mixing device described below. One of the subjects was familiar with the specific purposes of the study; the other 2 knew only that ME decay was to be assessed under various conditions.

Apparatus and Stimuli. The subjects sat facing a piece of acrylic rear-projection material mounted on a black background. The induction, interference, and test patterns appeared on this surface as circles $23^{\circ}$ in diameter.

All patterns were constructed on slides shown with Kodak Carousel slide projectors. Those used to induce MEs were made with Kodak Wratten filters No. 53 (green) and No. 32 (magenta); a neutral-density filter (Wratten No. 96) was used with No. 32 to make the luminances of the two stimuli more similar. When the Wratten filters were combined with 1.6-cpd gratings, the resultant patterns had space-averaged luminances of $147 \mathrm{~cd} / \mathrm{m}^{2}$ (green) and $130 \mathrm{~cd} / \mathrm{m}^{2}$ (magenta).

The slides used to produce the interference effect were prepared from the same grating pattens described above. After neutral-density filters (Kodak Wratten No. 96) were added to the gratings, their space-averaged luminance was $178 \mathrm{~cd} / \mathrm{m}^{2}$.

The stimulus used for ME assessment was a center/surround arrangement. The surround comprised gratings that had the same spatial frequency as the induction and interference patterns; the gratings were oriented vertically on the top half and horizontally on the bottom half. The center was an unlined circular area $7.5^{\circ}$ in diameter. A homogeneous field of variable chromaticity was projected onto this portion of the screen; the subjects adjusted its appearance to match that of the MEs seen on the adjacent gratings. The source of this field was a projection colorimeter (modeled after a design by Riggs, 1964), which mixed light transmitted by two Kodak Wratten color-compensating filters (No. CC3OM and No. CC50G). Their spectral transmissions, together with the spectral characteristics of the projector components, were used to calculate the loci of these two filters in CIE color space, with the resultant coordinates being $x=.421, y=.350$, and $x=.407, y=.454$, respectively. All mixtures of the two filters can be located in CIE space on a straight line that connects these two points, according to the proportion contributed to a particular match by each filter.

Absorbing-glass neutral-density filters (Corion Corp.) were used to attenuate the intensities of both portions of the test stimulus (lowluminance test patterns are associated with strongest assessed MEs; see White, 1976). The space-averaged luminance of the lined portion of the test pattern was $4 \mathrm{~cd} / \mathrm{m}^{2}$. The luminance of the matching spot was $8 \mathrm{~cd} / \mathrm{m}^{2}$. These filters also were used to produce an $8-\mathrm{cd} / \mathrm{m}^{2}$ achromatic homogeneous field used for light adaptation purposes, and to produce a second achromatic homogeneous field used as a matched-luminance $\left(178 \mathrm{~cd} / \mathrm{m}^{2}\right)$ control for the achromatic interference gratings.

Procedure. Each session began with $1 \mathrm{~min}$ of light adaptation. Then the test pattern was presented, and the subjects made one match to the top and then one to the bottom portion of the pattern. These matches were used only to confirm the absence of residual effects from previous sessions.

Preinduction interference was produced by having the subjects view horizontal and vertical achromatic gratings that alternated every $10 \mathrm{sec}$ for $5 \mathrm{~min}$. In control sessions, the subjects viewed an achromatic homogeneous field for $5 \mathrm{~min}$. If the condition involved a delay between the interference pattern exposure and $\mathrm{ME}$ induction, there was a time period during which the subjects left the laboratory and went about routine business for a set interval (for 2 subjects the interval was $15 \mathrm{~min}$; for 1 it was $7 \mathrm{~min}$ ). At the end of this interval, or immediately after exposure to the achromatic stimulus in the no-delay conditions, the ME was induced by having the subjects view the two chromatic gratings alternating every $10 \mathrm{sec}$ for $5 \mathrm{~min}$. After ME induction, there was a 1 -min period of light adaptation, and then the test slide reappeared and its two halves were matched. The light-adaptation and match sequence was repeated four times at $1 / 2-h$ intervals.

Each subject participated in four sessions per condition, for a total of 16 sessions per subject in a 2 (grating vs. control) $\times 2$ (immediate vs. delay) factorial design. In addition to this basic design, 2 subjects participated in three extra sessions for the grating/15min delay cell. In these extra sessions, the subjects remained in a dark room during the delay interval.

Two to 4 days elapsed between sessions, and the colororientation combination in the ME stimuli was reversed between sessions.

\section{Results}

For each ME assessment, matches to the two portions of the test pattern were converted to CIE coordinates. A simple index of ME strength was taken to be the distance in CIE space between loci for the two matches.

Figure 1 shows a summary of the results, comparing the grating and the control conditions for no delay, 15min delay, and 7-min delay between the achromatic exposure and the ME induction. When there was no delay (bottom graph), the ME was weaker in the grating condition than in the control condition by about the same 

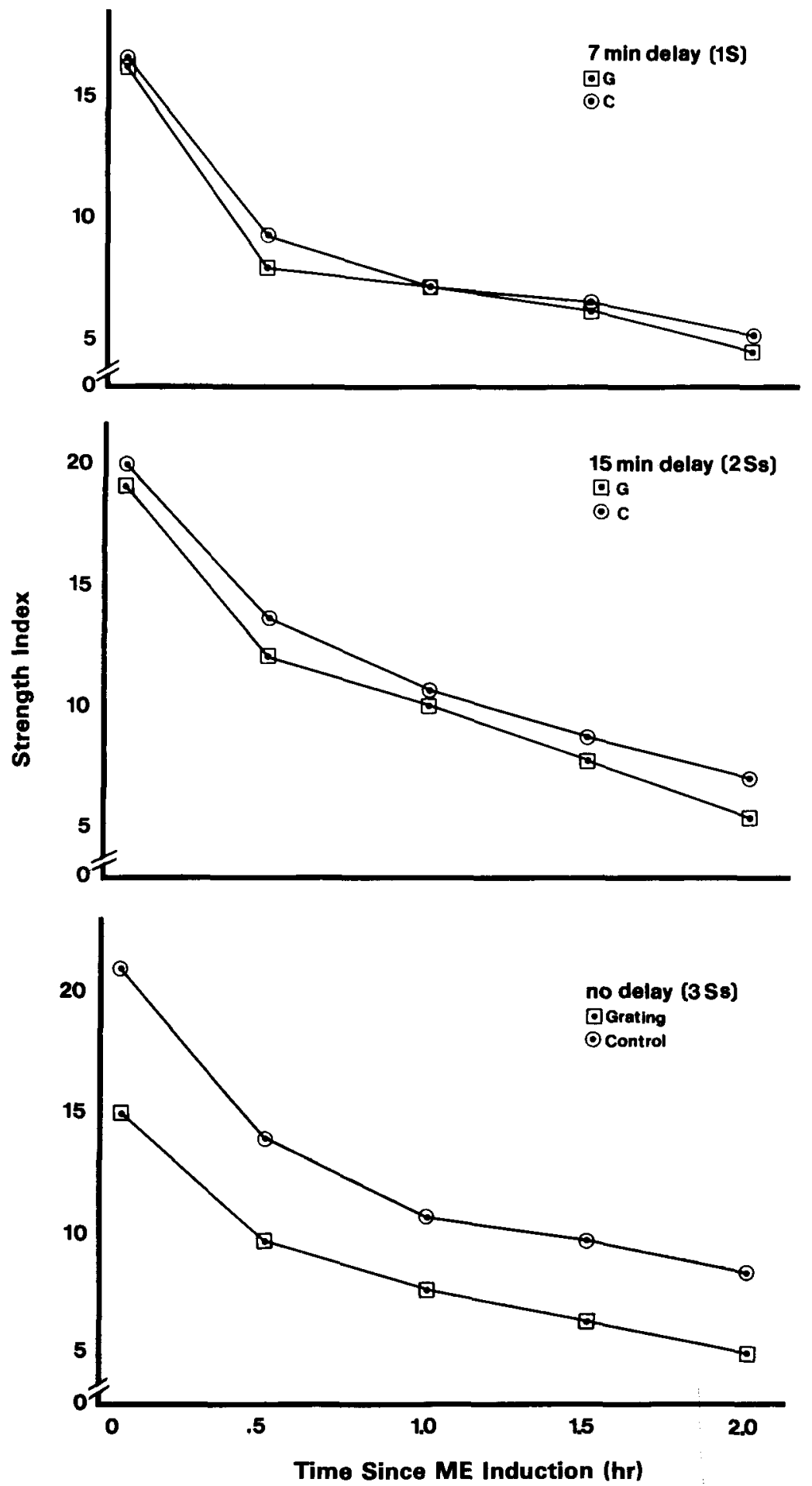

Figure 1. The strengths of McCollough effects (MEs) subject to preinduction interference are shown as a function of time since induction. The bottom graph depicts results from conditions in which $\mathrm{ME}$ induction immediately followed the interference stimuli (square symbols) or control stimuli (circular symbols); data from all 3 subjects are combined. The middle graph shows results from the 2 subjects who had a 15-min delay between the $\mathrm{ME}$ and preinduction stimuli; the top graph shows results from the 1 subject who had a 7-min delay. 
amount throughout the 2-h testing period. Two-way (observer $\times$ condition) analyses of variance (ANOVAs) applied to the data at each test point revealed highly significant differences $[F(1,9)=52.6,37.2,12.6,18.2$, and 23.8 , for the successive test points; all $p \mathrm{~s}<.01$ ] between the two conditions at all test points.

In both of the delay conditions (middle and top graphs), however, the MEs in the two conditions were very similar throughout the testing period. ANOVAs applied to these data revealed no significant differences at any test points.

Data from the extra sessions in which the subjects remained in darkness during the 15-min delay between grating exposure and ME are not shown; this curve was indistinguishable from the two curves depicted in the middle graph of Figure 1.

\section{Discussion}

The results of Experiment 1 confirm and extend previous reports of preinduction interference. When subjects view achromatic gratings immediately before they are exposed to the ME induction patterns, the resultant ME is depressed relative to the control ME. Furthermore, as was reported by White and Graves (1976), the grating and control decay curves are parallel; at least over the 2-h testing period, there is no sign that the effect of the grating inspection dissipates. Rather, it is as if the resultant ME were simply an unusually weak one-about $70 \%$ of control strength, in this case-that decayed naturally.

The unanticipated and possibly most important result of Experiment 1 is that preinduction interference does not occur if there is some delay between exposure to the achromatic gratings and the ME induction. Whatever the process by which achromatic gratings interfere with subsequently induced MEs, it apparently does not operate for any length of time, even in darkness.

\section{EXPERIMENT 2}

Experiment 2 was designed to describe postinduction interference in a paradigm similar to that used by White and Graves (1976).

\section{Method}

The participants, apparatus, and stimuli were the same as in Experiment 1; however, the procedure differed in order to produce postinduction interference. After the initial matches to the test pattern, the subjects viewed the alternating chromatic stimuli for $5 \mathrm{~min}$; then they light-adapted for $1 \mathrm{~min}$, and subsequently matched the two halves of the test slide. Next, they viewed either the alternating achromatic gratings or the achromatic homogeneous field for $5 \mathrm{~min}$, and then again light-adapted and matched the test stimulus. The light-adaptation and test-matching sequence was repeated eight more times, first at $1 / 2-h$ and then at $1-h$ intervals from the first postME match. An entire session spanned about $6 \mathrm{~h}$.

Each of the 3 subjects participated in four sessions per condition, making eight sessions in all per subject. At least 2 days elapsed between sessions, and the color-orientation combination in the $\mathrm{ME}$ stimuli was reversed in successive sessions.

\section{Results}

ME strength was assessed in the same manner as described for Experiment 1. Initially, MEs in the grating condition were almost identical to those in the control condition; after the achromatic exposure, they were weaker at the second and third test points. At the remaining seven test points, however, MEs in the two conditions were highly similar. Figure 2 shows the data from the first $2 \mathrm{~h}$ of Experiment 2 . Two-way (observer $\times$ condition) ANOVAs applied to the data at each test point revealed significant differences between conditions only for the second $[F(1,9)=9.6, p<.02]$ and third $[F(1,9)=$ $7.1, p<.03$ ] tests. Tests at $1 \mathrm{~h}$ or more after ME induction were not significantly different in the two conditions.

\section{Discussion}

In Experiment 2, MEs subjected to postinduction interference showed an initial depression, in which they were assessed at approximately $80 \%$ of control strength. However, the interference effect was rather short-lived; the grating and control curves converged within about $1 \mathrm{~h}$ of induction.

There was little evidence of recovery-that is, an increase in ME strength-in these data. Instead, the grating curve dropped first faster and then slower than the control curve to ultimately join it. This result was unexpected, given past evidence of strength gains in White and Graves's (1976) and Skowbo and Clynes's (1977) studies. Examination of the methodology in these earlier papers suggests two possible reasons for the discrepancy. First, subjects in Skowbo and Clynes's study kept the adapted eye in the dark during the rest period, whereas subjects in the present Experiment 2 were in a lighted environment between assessments; perhaps darkness is necessary

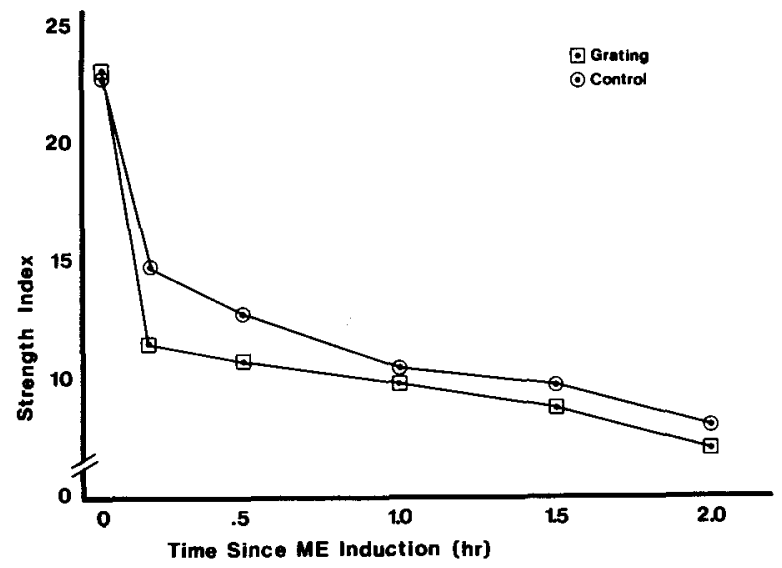

Figure 2. The strengths of McCollough effects (MEs) subject to postinduction interference are shown as a function of time since induction. Data from the 3 subjects are combined. Square symbols indicate exposure to interference stimuli; circular symbols indicate the control stimuli. Achromatic stimuli were presented immediately after the first ME assessment. 
for a revival effect to occur. Second, subjects in both of the previous papers were tested more frequently than those in the present Experiment 2; perhaps a strength increase did occur in the present experiment, but subsequent decay already had begun by the time the tests occurred.

\section{EXPERIMENT 3}

Experiment 3 was designed to investigate possible reasons for the discrepancy between results of Experiment 2 and previous work demonstrating increases in ME strength following the initial depression of postinduction interference. It was essentially a 2 (grating vs. control) $\times 2$ (intertest intervals spent in light vs. dark) factorial design, with tests carried out more frequently than in Experiment 2.

\section{Method}

Subjects. Two females participated. One had been a subject in the previous two experiments; the other had never participated in ME experiments and was naive as to the goals of this study. Her color-discriminating ability was also in the superior range, as assessed by the Farnsworth-Munsell 100-Hue Test.

Apparatus and Stimuli. The apparatus and stimuli were basically the same as in Experiments 1 and 2. However, a routine replacement of lamps and filters prior to the start of Experiment 3 resulted in slightly different luminance values for the various stimuli. The new values were: green grating, $158 \mathrm{~cd} / \mathrm{m}^{2}$; magenta grating, $137 \mathrm{~cd} / \mathrm{m}^{2}$; achromatic gratings and achromatic homogeneous control stimulus, $171 \mathrm{~cd} / \mathrm{m}^{2}$; light adaptation field, $6.1 \mathrm{~cd} / \mathrm{m}^{2}$; test grating, $3.4 \mathrm{~cd} / \mathrm{m}^{2}$; matching spot, $7.5 \mathrm{~cd} / \mathrm{m}^{2}$.

Procedure. The procedure was the same as in Experiment 2, except that the testing schedule was compressed. Tests subsequent to the one immediately after achromatic inspection were taken every $15 \mathrm{~min}$ following the initial ME (rather than every $30 \mathrm{~min}$ ), to $1 \mathrm{~h}$ after $\mathrm{ME}$ induction. Each subject participated in four runs per cell of the $2 \times 2$ design, or 16 runs per subject.

\section{Results}

ME strength was assessed in the same manner as described previously; results are shown in Figure 3. Examination of the figure reveals that, overall, the MEs did show a more rapid decline in light than in darkness. Threeway (observer $X$ condition $\times$ interest lighting) ANOVAs applied to data at each test interval indicated that the light and dark pairs of curves had diverged significantly $[F(1,6)$ $=8.4, p<.03$ ] at $15 \mathrm{~min}$ after ME induction; they remained apart by a comparable or greater amount throughout the $1 \mathrm{~h}$ or so of testing.

Postinduction exposure to the achromatic gratings produced a significant initial depression relative to the control conditions $[F(1,6)=31.0, p<.01]$; then, the grating and control curves converged in one of two patterns, depending on whether the intervening time had been spent in the light or in the dark. The curves for the light conditions generally replicate the results of Experiment 2; the grating curve dropped rapidly and then more slowly to meet the control curve. In both experiments, the curves merged by the third assessment following achromatic exposure; however, the convergence occurred somewhat

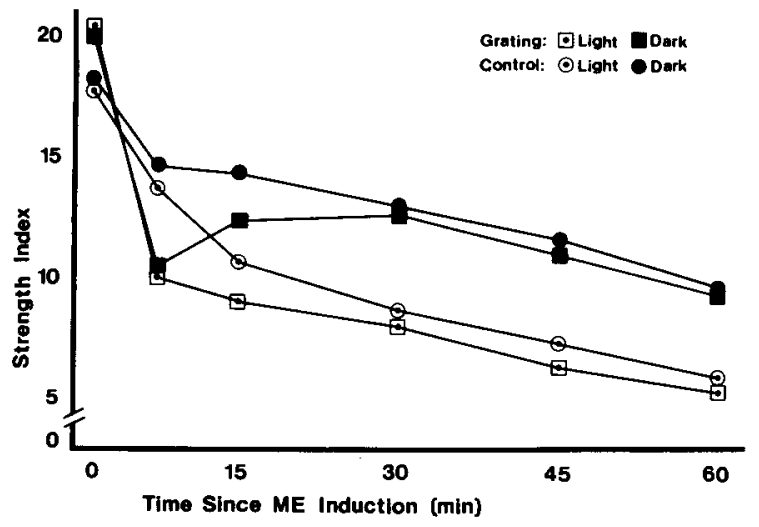

Figure 3. The strengths of McCollough effects (MEs) subject to postinduction interference are shown as a function of time since induction for two intertrial lighting conditions. Open symbols represent conditions in which subjects remained in the light between tests; closed symbols represent conditions in which subjects were in darkness. Data from the 2 subjects are combined. Square symbols indicate exposure to interference stimuli; circular symbols indicate the control condition. Achromatic stimuli were presented immediately after the first ME assessment.

sooner in Experiment 3 (within about $30 \mathrm{~min}$ ) than in Experiment 2 (within about $1 \mathrm{~h}$ ).

In the dark conditions, ME strength showed a revival following its initial decline. The grating curve appeared to be catching up with the control curve at about the same rate as in the light conditions; however, because the control curve had fallen off at a relatively slow rate, an increase in assessed strength affected the convergence.

\section{Discussion}

The results of Experiment 3 are similar to those of Experiment 2 with regard to the resolution of postinduction interference: the grating decay curve ultimately joins the control curve. The results of Experiment 3 also resolve the discrepancy between Experiment 2 and Skowbo and Clynes's (1977) study. Recovery from postinduction interference is more likely to show an increase in assessed strength following the initial depression when the decay periods are spent in darkness, possibly because control decay is relatively slow under these conditions.

The difference in the decay characteristics of the two control conditions contradicts results of an earlier experiment by Skowbo et al. (1974), who found no difference in decay rates of MEs in darkness and in various stimulation conditions. However, as noted by MacKay and MacKay (1975) in their paper on ME storage in darkness, the long light-adaptation periods in the Skowbo et al. experiment may have undermined a comparable phenomenon. The results of Experiment 3 support MacKay and MacKay's findings and their interpretation of Skowbo et al.'s results; light-adaptation periods were very short in the present experiment.

Taken together, the results of Experiments 2 and 3 enable me to describe the effect of achromatic inspections 
on prior MEs. Two important characteristics emerge. First, the depression in strength is temporary, lasting less than $1 \mathrm{~h}$ (variations may depend on the testing schedule). Second, after the initial depression, ME strength attains the level it would have had if not for the inspection of achromatic contours, and it remains at that level over its subsequent decay.

These results generally confirm those reported by White and Graves (1976). However, the data they presented suggested that the influence of achromatic grating inspection was fairly prolonged; a ME that had recovered from postinduction interference (and begun to decline again) seemed to remain reliably weaker than the MEs in the comparison decay curve. Perhaps this discrepancy can be explained by the nature of the comparison condition (an explanation that raises the possibility that the control stimulus affected decay rate). On the other hand, the interference curve shown by White and Graves (1976) describes only one run for one subject. Interference curves for other subjects, available in Graves (1976), show more of a tendency to converge with the control line; thus the present results seem reasonably consistent with the previous ones.

\section{EXPERIMENT 4}

Experiment 4 was designed to determine whether any recovery from preinduction interference could be observed if decay were tracked in the dark, as was done following postinduction interference in Experiment 3.

\section{Method}

The subjects in Experiment 4 were a female who had served in Experiments 1-3 and another female who had not participated in any of the previous experiments. The second subject was naive as to the purpose of the experiment; her color-discriminating ability was also in the superior range.

The apparatus and stimuli were the same as in Experiment 3. The procedure was identical to that of Experiment 1 except for the testing schedule and the nature of the intertrial intervals. In Experiment 4, tests were carried out immediately after ME induction, and then every $15 \mathrm{~min}$ for a total of four postinduction tests. The subjects

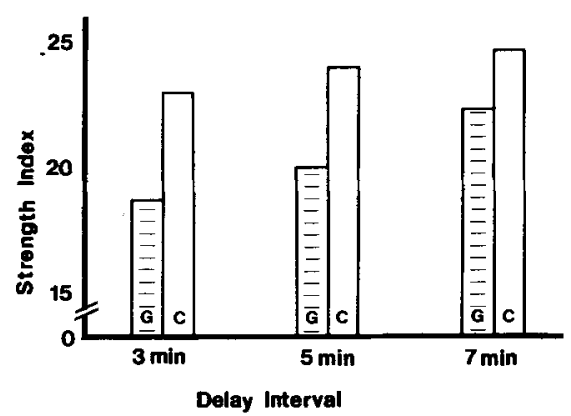

Figure 4. The initial strengths of McCollough effects (MEs) subjected to preinduction interference are shown as a function of three delay intervals between preinduction exposure and ME induction. Data from the 2 subjects are combined. At each delay interval, the hatched bar represents the interference condition and the open bar represents the control condition. remained in darkness between tests. Each participated in four runs of the grating condition only.

\section{Results and Discussion}

ME strength was assessed in the same manner as described previously. A plot of the results of Experiment 4 was highly similar in form to the initial hour of the immediate-grating curve of Experiment 1. There was no increase in ME strength, either in the averaged results or in any of the individual runs. These results are consistent with those of Experiment 1; there is no sign of recovery from the effects of preinduction interference, even when intertrial intervals are spent in darkness.

\section{EXPERIMENT 5}

A fifth experiment was designed to determine whether preinduction interference could be obtained with even shorter delay times than those used in Experiment 1.

\section{Method}

The subjects, apparatus, and stimuli were the same as in Experiment 4 . The elements of the procedure were the same as in previous experiments, arranged in Experiment 5 to include a pretest, a 5-min period of exposure to either gratings or control stimulus, a delay period during which the subject remained in darkness, a 5-min ME induction period, and a single ME assessment. Three delay periods were used: $7 \mathrm{~min}, 5 \mathrm{~min}$, and $3 \mathrm{~min}$. Each subject participated in four runs per condition, or 24 runs in all.

\section{Results}

ME strength was assessed as in Experiments 1-4. The results are shown in Figure 4, which reveals that, as time separating the ME from preinduction stimulation increases, the differences between the control and grating condition decrease. Two-way (observer $\times$ condition) ANOVAs applied to data at the three delay intervals showed a highly significant difference between the two conditions at the 3 -min delay $[F(1,6)=34.9, p<.01]$, and nonsignificant differences at the 5-min $[F(1,6)=5.5$, $p<.06]$ and $7-\min [F(1,6)=1.3]$ delays.

\section{Discussion}

The results of Experiment 5 confirm and extend those of Experiment 1. The effects of preinduction interference are very short-lived, becoming negligible if the time period between grating exposure and ME induction is more than approximately $5 \mathrm{~min}$. Thus, whereas postinduction interference can operate on a prior ME for at least as long as $\mathbf{1 0 0} \mathrm{min}$ after induction (White \& Graves, 1976), preinduction interference can operate on a subsequent ME only if ME induction follows the grating stimuli within a few minutes.

\section{GENERAL DISCUSSION: THEORETICAL IMPLICATIONS}

According to the classical conditioning approach to MEs, lines in a particular spatial configuration serve as a conditioned stimulus. If interference effects are inter- 
preted accordingly, then postinduction interference is viewed as extinction and any subsequent revival as spontaneous recovery, and preinduction interference is seen as analogous to latent inhibition. Results of the present experiments argue most strongly against this view of preinduction interference: preinduction interference occurs only if the gratings are viewed within a few minutes of the induction stimuli; this finding stands in contrast to those in the conditioning literature. According to studies reviewed by Lubow (1973), latent inhibition occurs even with delays as long as $48 \mathrm{~h}$ between the nonreinforced preexposures to the conditioned stimulus and the beginning of acquisition.

It may be argued that the observation of gains in strength only with dark intertrial intervals supports the spontaneous recovery position; darkness may provide more of a rest for the visual system than does a lighted environment. However, in looking at the results of Experiments 2 and 3 , it seems that recovery from postinduction interference is most simply described as a convergence of the interference decay curve with a control curve: the ME returns to the strength it would have had but for exposure to the contours. A gain in strengh following interference is most likely to be observed when the control ME is particularly strong.

Another interpretation of interference effects has been that exposure to achromatic gratings generates another pattern-contingent aftereffect (or group of aftereffects) that competes or summates with the ME. Results of the present experiments do not support this position. White and Graves (1976) found that postinduction interference effects could be observed for well over an hour following the chromatic inspections; however, the present results indicate that preinduction interference can be observed only with delays of a few minutes or less between the achromatic and chromatic inspections. Thus, if interference results from the operation of another contingent aftereffect, it is one that decays much faster than standard chromatic MEs; it would also be unlike MEs in that its decay is not retarded in darkness.

A third interpretation of interference effects is Graves's (1976) suggestion that inspecting achromatic gratings makes subsequently viewed patterns less effective as stimuli. This position, an outgrowth of work on visual channels selective for features such as spatial frequency and orientation (see, e.g., Braddick, Campbell, \& Atkinson, 1978), was based on a phenomenon investigated by Blakemore, Muncey, and Ridley (1973)-namely, that inspecting high-contrast grating patterns produces an aftereffect of reduced perceived contrast of patterns similar in spatial features to the adaptation pattern (a simple, not contingent, aftereffect). Among other things, Blakemore et al. determined the magnitude and time course of the contrast reduction effect as a function of time spent adpating to the high-contrast pattern. The initial magnitude of the effect increased with longer adaptation times, up to a maximum after about $45 \mathrm{sec}$ of adaptation; longer inspection times did not produce a greater aftereffect. However, recovery time did continue to increase with longer exposure periods; it took about $6 \mathrm{~min}$ for the subject to recover completely from $5 \mathrm{~min}$ of adaptation.

This time course of recovery is consistent with the time course of preinduction interference in the present experiments. Also, it has been shown that MEs become weaker as the contrast of the inspection patterns decreases (Ellis, 1977). The missing link is evidence that a reduction in perceived contrast of the inspection patterns would have the same impact on MEs as a reduction in their actual contrast.

A similar problem exists with regard to the hypothesis that postinduction interference occurs because the apparent contrast of the test pattern is reduced; the available evidence (White \& Graves, 1977) concerns a reduction in actual contrast. In addition, however, the contrast reduction idea seems less applicable to my results than to those of Graves (1976) because I find that the time courses of the two phenomena are not consistent. Had apparent contrast reduction of subsequently viewed test patterns been the reason for the reduced ME strength, then recovery should have occurred sooner than it did.

According to the foregoing discussion, the results of the present experiments failed to support any of the major explanations for interference effects. Of the three, the contrast reduction account is the only one that attempts to relate interference effects to other established perceptual phenomena, and there may be some merit in pursuing that line of reasoning. Viewing a high-contrast grating for several minutes produces several pattern-selective adaptation effects, which are usually explained in terms of fatigue of the neural mechanism that processes that grating's features. Speculations that the ME might result from chromatic adaptation in edge detectors have been in the literature since McCollough's (1965) paper, yet there have been almost no attempts to link achromatic and chromatic aftereffects in the context of a neural model of MEs.

An exception to this theoretical gap is Grossberg's neural network architecture (see, e.g., 1987), which models various aspects of form and color perception, including MEs. According to this model, exposure to chromatic edges depletes or habituates the transmitter in a coloropponent contour channel. This imbalance is coded in a long-term memory trace, which decreases and thereby gates the signal in the habituated pathway. Thus, an achromatic edge, which initially generates equal signals in the opponent pathways, ultimately produces a gated signal that is larger in one pathway than in the other. The long duration of MEs is explained by the ability of the trace change to remain after transmitter habituation recovers. If that achromatic edge is repeatedly presented (as in a postinduction interference procedure), there will be equal transmitter habituation in both channels and the memory traces will equalize to produce equal gating in the oppo- 
nent channels, extinguishing the ME. It remains to be seen, however, how this model handles preinduction interference and recovery from postinduction interference.

In summary, an explanation of interference, or a theory of MEs that incorporates interference effects, must account for the following: First, the effect of postinduction interference can be described as a temporary disruption of an ongoing process; ME decay ultimately returns to its normal course. However, the dampening effect of preinduction interference is evident throughout the duration of the ensuing ME. Second, whereas the effect of achromatic gratings on a prior ME can be observed for as long as an hour, the ability of achromatic gratings to affect subsequently induced MEs lasts for only a few minutes. Although pre- and postinduction interference arise from exposure to the same stimuli, their dissimilar characteristics suggest the possibility that they are produced by different mechanisms.

\section{REFERENCES}

Allan, L., \& Siegel, S. (1986). McCollough effects as conditioned responses: Reply to Skowbo. Psychological Bulletin, 100, 388-393.

Blakemore, C., Muncey, J., \& Ridey, R. (1973). Stimulus specificity in the human visual system. Vision Research, 13, 1915-1931.

Braddick, O., Campbell, F., \& Atkinson, J. (1978). Channels in vision: Basic aspects. In R. Held, H. Leibowitz, \& H.-L. Teuber (Eds.), Perception: Volume VIII. Handbook of sensory physiology (pp. 3-38). New York: Springer-Verlag.

ELuIS, S. (1977). Orientation selectivity of the McCollough effect: Analysis by equivalent contrast transformation. Perception \& Psychophysics, 22, 539-544.

GRAVEs, A. (1976). Influence of viewing achromatic gratings on McCollough effects. Unpublished senior honors thesis, Brown University, Providence, RI.

GrossberG, S. (1987). Cortical dynamics of three-dimensional form, color, and brightness perception: II. Binocular theory. Perception \& Psychophysics, 41, 117-158.
Lubow, R. (1973). Latent inhibition. Psychological Bulletin, 79, 398-407.

MacKAY, D., MACKAY, V. (1975). What causes decay of pattern contingent chromatic aftereffects? Vision Research, 15, 462-464.

McCollough, C. (1965). Color adaptation of edge detectors in the human visual system. Science, 149, 1115-1116.

RigGS, L. (1964). A projection colorimeter. American Journal of Psychology, 77, 129-134.

Riggs, L., White, K., Eimas, P. (1974). Establishment and decay of orientation-contingent aftereffects of color. Perception \& Psychophysics, 16, 535-542.

SAvoY, R. (1984). "Extinction" of the McCollough effect does not transfer interocularly. Perception \& Psychophysics, 36, 571-576.

SHUTE, C. (1979). The McCollough effect: An indicator of central nerve transmitter activity. Cambridge: Cambridge University Press.

Skowbo, D. (1979). Luminance as a factor in the ability of achromatic gratings to interfere with McCollough effects. Perception \& Psychophysics, 26, 105-107.

SкоWво, D. (1984). Are McCollough effects conditioned responses? Psychological Bulletin, 96, 215-226.

SKOWBO, D. (1986). McCollough effects as conditioned responses? Reply to Allan and Siegel. Psychological Bulletin, 100, 394-397.

Skowbo, D., \& Clynes, N. (1977). Decline and revival of McCollough effects following inspection of achromatic gratings. Perception \& Psychophysics, 21, 180-182.

Skowbo, D., Gentry, T., Timney, B., \& Morant, R. (1974). The McCollough effect: Influence of several kinds of visual stimulation on decay rate. Perception \& Psychophysics, 16, 47-49.

WHITE, K. (1976). Luminance as a parameter in establishment and testing of the McCollough effect. Vision Research, 16, 297-302.

White, K., \& Graves, A. (1976, April). Influence of achromatic inspection on McCollough effects. Paper presented at the meeting of the Association for Research in Vision and Opthalmology, Sarasota, FL.

WhITE, K., \& GRAVES, A. (1977). Test pattern contrast influences assessed strengths of McCollough effects. Unpublished manuscript.

WhITE, K., \& SxowBo, D. (1980). Achromatic patterns influence McCollough effects: Altered perception of saturation? Unpublished manuscript.

(Manuscript received January 21, 1988; revision accepted for publication May 10, 1988.) 